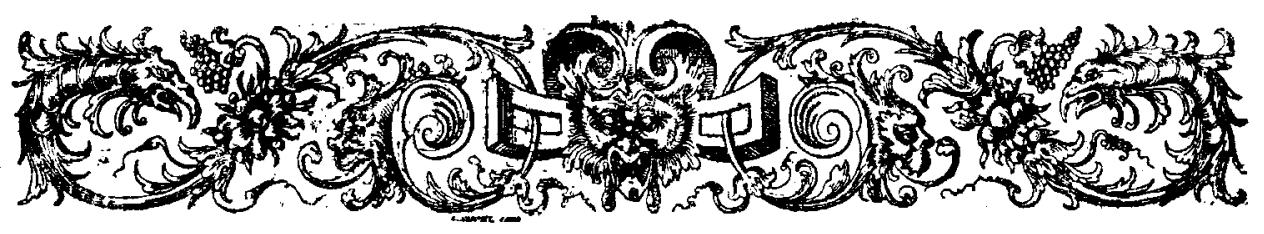

\title{
Nieuwe Gegevens omtrent Jacob Backer
}

\author{
MEDEGEDEELD DOOR
}

J. D. WAGNER.

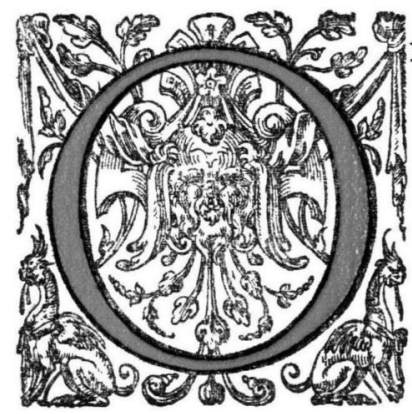

NDER eenige papieren betrekkelijk de familie VAN LOCKHORST trof ik de navolgende inventaris aan:

Inventaris van de goederen toebehoorende d' kinderen van salige DirRick BaCker en Sytie SchelLINGWOU saliger.

$\mathrm{A}^{0}$ 1653. In Amsterdam den 26 April.

Daaruit neem ik niet over wat uitgetrokken staat.

"Voor Claes BaCker, voor AnNa BaCkERs, voor ADRIAEN BACKER, voor d'kinderen int gemeen, evenmin de "Gedruckte boeken", maar van belang is de opgave der: „Schilderijen als volght."

I een diana met enige naeckte vrouties van.......... JACOB BACKER

2 een diana met boogh en pylkooker.............. dito

3 een mans troenie........................ dito

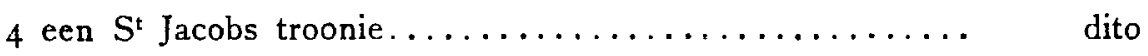

5 een op kykende mans troenie................. dito

6 een vrouwe tronie met een pluijm op............. dito

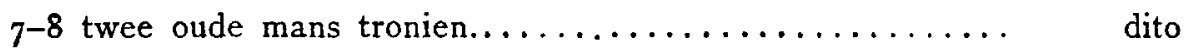

9 daer Eneas d' wapenen van Veenus ontf(angt) ........ dito

Io een pool met een poolze haemer............... dito

I I-I 4 vier cont(er)feyts(els) als 2 van D. B. en 2 van S. B.... dito

I5 een troonie van ClaAs BaCker............... dito

16-18 drie tekeninge als 2 groote en I kleene met glasen daer voor dito 
Ig een teekeningk van $D$. B. selfs glas voor.......... JACOB BACKER

20 een tronie van salige JACOB BACKEk van .......... Wyb. D'GeEST

2 I een conterfeijtsel van JAN BACKER.

23 een doot kintie van..................... A. V. Tempel

23 een lantschap met eenige beelties en paerties van........ S. v. GooR

24 een dito met een brugh met beesies.............. dito

25 een dito met een groote boom en bockies............. dito

26 een dito met enige swemmers.................. dito

27 een dito met eenige schaepies en beelties........... dito

28 een Maria Joseph en kintie van............. Thomas KeIJSER

29 een conterfeitsel van saligen JACOB BACKER (gegrav. d.

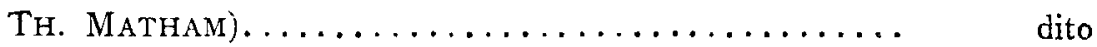

30 een oude mans tronie van $\ldots \ldots \ldots \ldots \ldots \ldots \ldots \ldots \ldots \ldots$ Strijker

3 I een lantschap met enige vrouwe beelties.............. dito

32 een dito met enige beelties en schaepies............. dito

33 een meysies tronie $\operatorname{van} . \ldots \ldots \ldots \ldots \ldots \ldots \ldots \ldots \ldots$ JAN LIEVENS

34 een schuertien met pap eeters............. Adriaen van Ostade

35 een schansie met een water met scheepies......... JAN VAN Goyer

36 een lantschap met een huysie en vissers ........... dito

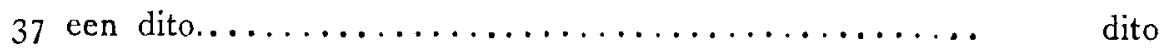

$3^{8}$ een harderins tronie AdAm Cameradius.

39 een tafel met een haes en ander wilt $\ldots \ldots \ldots \ldots \ldots \ldots \ldots$ E. VoNCK

40 een lantschap met een vallent water en groot gebou .... B. BREENBERG

4 I een ruwijn met een groot gebou en sluys........... dito

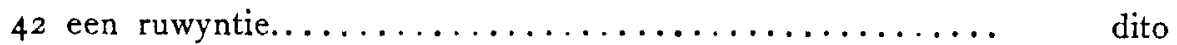

43 een onstuymige see.................... S. D' VILIEGER

44 Jaep hannes het $\mathrm{Y}$ en enige scheepen $\ldots \ldots \ldots \ldots \ldots \ldots$ dito

45 een lantschap met enige bockies ............. J. VAND ${ }^{\mathrm{R}}$ DOES

46 een mannetie en vroutie dat op d' veel speelt........ G. GeraErts

47 een Veenus met een slapende Cupedo.............. Stekade

48 een Narsissis...................... M. Uijtenkroeck

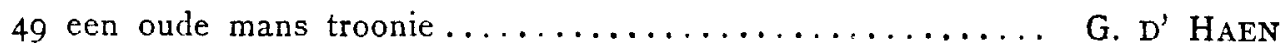

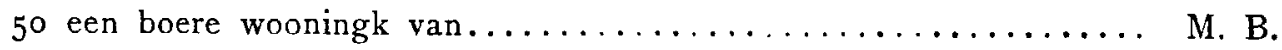

$5 \mathrm{I}$ een test met vier en roemer wyn van P. C. dooréén.

52 een biddende pannekoekebackster.

53 een holl met een schaepshooft, wortelen, cool van........ RyckHaLs

54 een holl met dito $\ldots \ldots \ldots \ldots \ldots \ldots \ldots \ldots \ldots \ldots \ldots \ldots$ dito

55 een slapent besie.

Oud-Holland 1922. 
56 een tronie met een vogeltie op d' hant.

57 d' distructie van Troye.

$5^{8 / 9}$ twee tekeningen van MOYAERT met glase daer voor.

60 een kerckie.

$6 \mathrm{I}$ een pannekoekebackster.

Volcht linnen; Allerleij lijwaet voor vrouwen te dragen; Luijermants goet. (Dit is niet overgenomen).

Dit voorstaende sijn die onvercofte goederen, berustende als blyct by de ondertekeninge.

i653 den i 5 Januw. vercoft aen TIERCK Jellemers

(voor een waarde van) $f \quad 230$

dito aen Femmetie Schellingh wou (voor een waarde van) $f$ i $3^{2}$

Van de vercofte inboel volgens d' Reeckennigh van de afslager

compt in suijver geldt.........................f $192 \mathrm{I}-\mathrm{I} \mathrm{I}$ -

Een kleen yser kacheltien, een schel en een mantelkas, saemen

aen $D^{r}$. VAN HENSBERGEN vercoft, samen voor...........f $\mathbf{2 2}$.

Dit voorenstaende is de Inventaris van de inboel en huysraet etc. int $t$ sterfhuijs van saliger Dirck Backer en Sijttien Schellinghwou bevonden; Berustende het onvercofte onder Femmetie Schellinghwou, ende tgeen vercoft is hebben wij ondergesc. gestelde voochden over de kinderen van gemelde overledene ten deele ontfangen en $t$ resterende sal in rekeninge goet gedaen werden. In kennisse der waerheijt hebben hier aff vier all eensluijdende gemaect waer van FEMmETIE SCHELlinghwoU, als oock d'ondergescr. voochden, ijder een tot haer genomen hebben. Aldus gedaen, in Amsterdam desen $3^{\circ}$ April Ao 1653

\section{TJERCK JELLEMERS SCHELLINGHER \\ TIERCK ABACKER \\ JACOB ROOLEEUW.}

Behalve dezen inventaris vond ik onder de Lockhorstpapieren nog eenige belangrijke genealogische notities, die mij aanleiding geven tot het opstellen van het nu volgende.

ADRIAEN TJERCKSE trouwt 7 Aug. i6i I Elsje Roelofs, zij overleed i 8 Aug. I614.

Hunne kinderen zijn: $\mathrm{I}^{\mathrm{e}}$ DIRK ADRIAENS BACKER, geb. I 8 Nov. 1612 en overl. 27 Nov. 1652. Hij trouwt 24 Aug. 1642 Sytie Schellingwou, d'r van Claas Janse Schellingwou en Anna Steffens. $Z_{i j}$ overl, 24 Mei 1652 in 't kraambed, oud $3^{2}$ en $2^{\circ}$ JUDICK ADRIAENS, geb. 3 Aug. I6I 4 en overl. 28 Maart I6 5.

ADRIAEN TJERCKSE moet uit een eerste huwelijk nog twee kinderen hebben gehad: Jacob Backer en TJerk Adr. Backer. De eerste omdat Dirk Adriaens 
BACKER in eene eigenhandige aanteekening zegt: „165 den 27 Augustij is myn broeder JACOB BACKER oover leeden sondagh smorgens ten ontrent 2 uyren." De tweede TJERK ADR. BACKER omdat hij blijkbaar zoon van een ADRIAAN is, dewijl hij voogd over de kinderen van DIRK ADRIAENS BACKER was en ingevolge een vroeger uitgegeven inventaris den 26 Maart 1659 dood moet zijn geweest. Toen leefden van zijne kinderen: LySBETH meerderjarig, JACOB, PIETER en ADRIAAN minderjarigen.

Het echtpaar BACKER-SCHELLINGwOU had tot kinderen:

ie. ElSIE BACKer, geb. I 8 Juni i643 en ob. 6 Sept. 1643.

2e. Claes Backer, geb. If Sept. I644, woog 24 weeken oud 23 pond. holl.

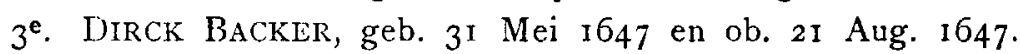

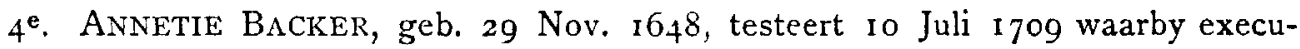
teurs DIRK VAN LOCKHORST en haar neef ADRIAAN BROUwER, ob. 29 Juli 1724 en 3 Aug. d. a. v. begr. in eigen graf $\mathrm{N}^{0} .4 \mathrm{I}$ in Noorderkerk te Amsterdam. Zij trouwt i 3 Sept. 167 I Nicol 1 AS VAN LOCKHORST, geb. 22 Mrt. 1640 en ob. I 7 Oct. I 7 .

Hunne kinderen volgen onder $A$ (opgave van den vader).

5. Adriaen BaCker, geb. I7 Mei 1652, overleden 26 Aug. I698 komende uit Oost-Indien den 9 dag dat hy in Zeeland gearriveerd was en is te Vlissingen begraven op den 2 Sept. oud zynde 45 jaar.

A. I. Lusya VAN Lockhorst, geb. I Juli 1672, ob. I Dec. I672; is begraven in de Noorderkerk int graf van ADRIAEN TIERCKSE van Harlingen daert merck op

2. Sytie v. L., geb. 26 Nov. 1673 , doopgetuigen JACOP VEEN en huisvrou en ob. I April 1676 .

3. Cornelis v. L., geb. $27 \mathrm{Mei}$ i675, doopgetuigen Dicrk Clase AbBas en Elisabet Backers en ob. 6 Febr. 1684.

4. DrRCK v. L., geb. 30 Dec. 1676 , doopgetuigen zyn freer ADRIAEN BACKER en syn huisvrou en ob. I 8 Oct. I 735 .

5. AdriaAn v. L., geb. ig Sept. i678, doopgetuigen zyn neef AdRiaEn BaCker en syn huisvrou Elsie Colijn, ob. I7 Oct I 716 en begr. in graf No. 4 I.

6. JACOP v. L., geb. 23 Jan. 1682, doopgetuigen JACOP BACKER en zyn huisvrou Elisabet Dootshooft en ob. 24 Sept. I682, begraven bij myne dochterkens.

7. Cornelis V. L., geb. I5 Juli i 684, doopgetuigen Jeremias Bosyn en Elisabet BACKers en begraven io Febr. I742.

8. JACOB v. L., 26 Sept. I686, doopgetuigen Pieter BaCker en Elisabet DOOTSHOOFT. 
9. Baertie v. L., geb. i A Aug 1688, doopgetuigen Dirck Classe Abbas en Luytie Jans syn huisvrou en ob. I4 Aug. 1688.

ro. Roeland v. L., geb. 3 Dec. 1689, doopgetuige AdriaAN Brouwer, ob. I I April 17 i 3 en begr. in eigen graf.

Uit het voorgaande kunnen wij nu besluiten dat de beroemde schilder JACOB BACKER tot vader had ADRIAEN TJERCKSE, een Harlinger. Zijn moeders naam blijft onbekend; zou het riet eene BACKER kunnen zijn? Omstreeks JACOB BACKER's tijd nemen ook de afstammelingen van de gebroeders CORNELIS JORISZ. alias ROODHOORN en JORIS JORISZ. alias ROODHOORN, onderscheidenlijk getrouwd met de gezusters Grietje en ANNETIE BACKER, den naam BACKER aan (de nu levende Jonkheeren BACKER). Kan de mededeeling van de namen der voogden in den inventaris van 1659 in deze wellicht licht brengen?

Eindelijk hebben wij thans de zekerheid dat JACOB BACKER de oom was van de gebroeders JACOB en ADRIAAN BACKER en vernemen wij dat de jongere JACOB met Elisabet DOOTShoOfT gehuwd was.

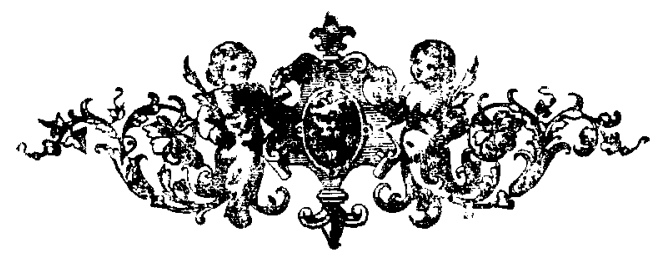

